\title{
Tubulopathies need not preclude living kidney donation: a case of successful transplantation from a donor with Gitelman syndrome.
}

\author{
Dr Daniel Stewart, Dr Dr Aisling Courtney, Dr Andrew Connor
}

\section{Introduction}

A 76-year old peritoneal dialysis patient with hypertensive nephropathy received an offer via the Kidney Sharing Scheme of a living kidney transplant from a 68-year old donor with an incidental diagnosis of Gitelman syndrome in 1995, but no other comorbidities. The donor was asymptomatic with normal serum potassium levels on $40 \mathrm{mg}$ amiloride daily. The offer was considered in the recipient centre's Transplant MDT. The benefits of a kidney from a living donor with lifelong low-normal blood pressure due to mild salt wasting were felt to outweigh the potential electrolyte abnormalities that might arise. The recipient was counselled about potential complications and the offer was accepted. Pre-transplant donor and recipient blood tests are listed in Figure 1.

\begin{tabular}{l}
\multicolumn{4}{c}{ DONOR } \\
\begin{tabular}{|l|l|l|l|}
\hline Creatinine & 88 & Creatinine & 723 \\
\hline Corrected Calcium & 2.83 & Corrected Calcium & 2.64 \\
\hline Phosphate & 0.75 & Phosphate & 1.81 \\
\hline Sodium & 137 & Sodium & 134 \\
\hline Potassium & 3.6 & Potassium & 5.1 \\
\hline
\end{tabular}
\end{tabular}

FIGURE 1. Pre-transplantation donor and recipient blood results.

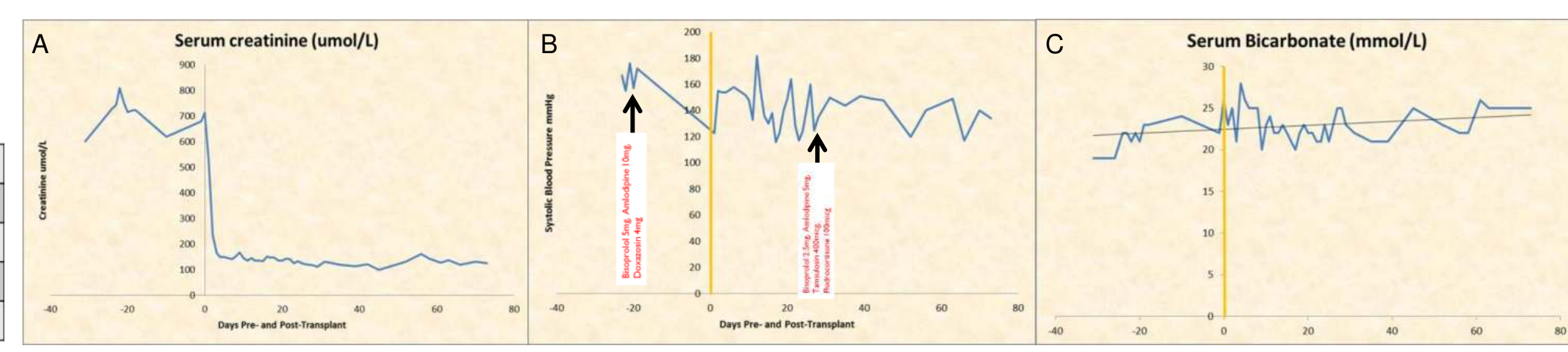

GRAPHS A-C: Pre- and post-transplantation measurement of serum creatinine in umol/L (A), preand post-transplantation random blood pressure measurements with corresponding blood pressure-lowering medication requirements $(B)$, and pre-and post-transplantation serum bicarbonate measurements with line of best fit $(C)$. Day 0 represents day of transplantation.

\begin{tabular}{l} 
Methods \\
$\begin{array}{l}\text { The standard immunosuppression protocol } \\
\text { (basiliximab/methylprednisolone, then tacrolimus, } \\
\text { mycophenolate and prednisolone) was adhered to. } \\
\text { The recipient's serum and urinary electrolytes were } \\
\text { closely monitored in the first eighty days of the } \\
\text { post-operative period in addition to routine } \\
\text { monitoring of blood pressure. }\end{array}$ \\
\hline
\end{tabular}

\section{Results}

Serum creatinine fell immediately to a baseline of $110-130$ umol/L. Serial serum and urinary electrolyte concentrations revealed transference of the classical Gitelman biochemical phenotype to the recipient: hypomagnesaemia with inappropriate renal magnesium losses; hypocalciuria and mild hypercalcaemia; a trend towards alkalosis; and, a reduction in serum potassium levels (although never below $3.0 \mathrm{mmol} / \mathrm{L}$ and not requiring supplementation). An unexpected profound hyponatraemia was probably multifactorial and resolved. There was a marked reduction in the recipient's antihypertensive medication requirement

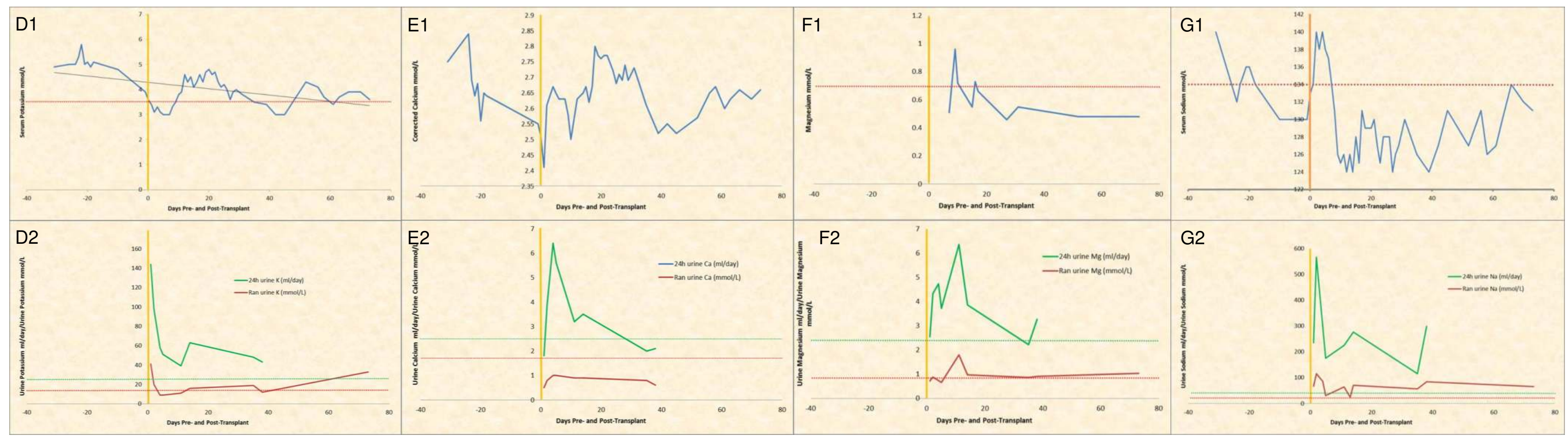

GRAPHS D1-G2: Pre- and post-transplant measurement of serum potassium (D1), spot and 24-hour urinary potassium (D2), serum calcium (E1), spot and 24-hour urinary calcium (E2), serum magnesium (F1), spot and 24-hour urinary magnesium, serum sodium (G1), spot and 24-hour urinary sodium (G2). Day 0 represents day of transplant. Dotted green lines represent lower limit of normal range for specific 24hour urinary electrolyte collection. Dotted red lines represent lower limit of normal range for specific serum/spot urinary electrolyte measurement

\section{Discussion}

This case demonstrates that tubulopathies need not preclude living donation biochemical abnormalities may be transferred but can be anticipated and treated. Furthermore, tubulopathies may in fact confer benefits.

In this case, firstly, the donor renal parenchyma had benefitted from lifelong low normal blood pressure as a consequence of the Gitelman syndrome and, secondly, the reduction in functional NCC receptors in the distal tubule of the donor kidney prevented their upregulation by tacrolimus and this might be anticipated to mitigate the drug's more common complications (renal tubular acidosis, hypertension, hyperkalaemia and hypercalciuria) and prevent toxicity.

\section{Learning Points}

- Tubulopathies need not preclude living donation and should not be considered an absolute contraindication.

- Transplantation from a donor with a known tubulopathy is likely to result in transference of the biochemical phenotype to the recipient.

- Transplantation from a donor with Gitelman syndrome may theoretically provide protection against the potential risk of tubular toxicity from CNI medication. 